\title{
A novel collective health monitoring of a wind park
}

\author{
Kritika Sodha, George Fernandez. S, Vijayakumar. K, Sattianadan. D \\ Department of EEE, SRMIST, Chennai
}

\begin{tabular}{l} 
Article Info \\
\hline Article history: \\
Received Jan 14, 2020 \\
Revised Mar 15, 2020 \\
Accepted May 3, 20 \\
\hline Keywords: \\
Health monitoring \\
Machine learning \\
Renewable power \\
Wind park \\
Wind power
\end{tabular}

Corresponding Author:

George Fernandez. S

Department of EEE

SRMIST, Chennai

Email: George.electrix@gmail.com

\begin{abstract}
Compared to a time-based maintenance schedule, condition-based maintenance provides better diagnostic information on the health condition of the different wind turbine components and subsystems. Rather than using an offline condition monitoring technique, which require the WT to be taken out of service, online condition monitoring does not require any interruption on the WT operation. The online condition monitoring system uses different types of sensors such as vibration, acoustic, temperature, current/voltage etc. Using a machine learning approach, we aim to establish a data driven fault prognosis framework. Instead of traditional wired communications, wireless communication systems such as wireless sensor network have the advantages of easier installation and lower capital cost. We propose the use of WSN for collecting and transmitting the condition monitoring data to enhance the reliability of wind parks. Using data driven approach the collective health of the WP can be represented based on the condition of the individual wind turbines, which can be used for predicting the remaining useful life of the system.
\end{abstract}

This is an open access article under the CC BY-SA license.

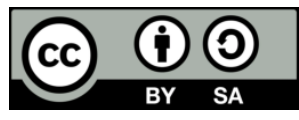

\section{INTRODUCTION}

The conventionally used maintenance scheduling involves time based scheduled maintenance which lack in providing proper information of health condition of equipment also maintenance is provided disregard of the its need. The work proposes a Predictive maintenance over scheduled/time based maintenance. As maintenance cost is a key portion of the total cost of electricity generated by WT work in this field is required to minimise it $[1,2]$. Compared to a time-based maintenance schedule, condition-based maintenance provides better diagnostic information on the health condition of the different WT components and subsystems. Due to the exposure to harsh environment wind turbine (WT) have high failure rates [3-5]. Due to lack of data, exact location of the fault is not detected. Not much attention is given to fault prognosis in literature. Conventional method of condition monitoring (CM) make use of vibration analysis which is not enough to gather the adequate information required for providing accurate predictive maintenance, as the signal acquired from the vibration sensors are prone to the environmental noise. Adding to this current signal can be safely collected at remote condition $[6,7]$. Combining current signature analysis (CSA) with vibration analysis will provide better information regarding the condition of WT subsystem on rotor asymmetry detection in WT suggested generator current signals to analyse asymmetries [8]. It makes use of EKF to figure out the fault signature component. The results shows that EKF when compared to continuous wavelet transform and iterative discrete Fourier transform promises low cost and efficiency for monitoring the output of WT. This also suggests how a CWT can't produce fine resolution in time and frequency domain, adding to this large time is required to compute it [9-12]. The IDFT and EKF take similar computational time which makes them 
suitable for online condition monitoring. In [13] the FP and RUL prediction of WT gearbox is proposed a Particle Filtering approach for prediction and the result also showed that ANFIS performed better than RNN for state transition of the fault feature. In this work ANFIS and RNN are used to train the generator for the state transition of fault feature. The particle filtering approach will then predict the RUL. The harmonic transient analysis of WT using extended harmonic domain model [14-16] suggested the use of DFIG as it allows only a portion of power to pass through the converter that leads to reduced size, cost and losses. The paper analyse harmonic frequency in the transient state. Any type of control scheme can be applied as it can be easily converted into time domain as it provides instantaneous time-variation of harmonics. A research [17] discuss about various advancement in predictive maintenance. They also discuss about the challenges and principles of using generator current signature analysis and collective condition monitoring for WT. they suggest extracting multiple fault features like SNR, kurtosis, RMS values and performing fault diagnosis with aid of machine learning methods. A data driven design for FD using RF and XGboost ensemble learning method is proposed in [18-20]. Combining these methods will increase the WT fault classifier efficiency. RF and XGboost have proven their efficiency and effectiveness in their classification and regression application. They use first-order filters to reduce the noise disturbances. A tree structure classifier is used so that the most dominant signals can be used as fault features. They use harmonic order tracking analysis method for improving the fault diagnosis reliability. Proposed methodology doesn't require speed measurements and the results are plotted similar to a fourier spectrum. They also reduced the parameters required to analyse the machine condition.

Multiclass SVM-based fault identification schemes using the time- and frequency-domain features are used and both stator and rotor current for a multisensory information fusion-bases FD and identification framework is used for WT health monitor [21]. Tasnim [22] proposed frequency analysis and a stacked auto encoder based multi class SVM deep classifier-based fault diagnosis using rotor current. Hilbert-transform was used for envelope extraction, and an angular resampling algorithm was developed to solve the spectrum smearing problem caused by shaft speed variations. Jiang, [23] in their work proposed denoising autoencoder with temporal information. Sliding-window technique is utilised so the Denoising Auto encoder captures nonlinear correlations among multiple variables and temporal dependencies at each variable. The research works [24-25] proposed multilevel dendritic cell algorithm-based FD and isolation technique. It also integrate time window for online FD strategy. It is also compared with negative selection algorithm based FD and identification techniques. The proposed method requires low computational complexity. Quantitatively better results are obtained by the proposed method.

\section{OBJECTIVE OF THE WORK}

The objective of the work can be broadly classified as the following points:

a) The shift from scheduled maintenance to condition based maintenance

b) Change in the electrical parameters is detected by current signature analysis which can be used to detect possible failures in the system to undergo diagnosis and prognosis.

c) By application of data driven approaches for condition based monitoring we are looking forward for a result that maximises the energy conversion by minimizing the damage caused by faults at the earliest with maximum accuracy and make our wind energy conversion system highly reliable and secured.

\subsection{Condition assessment using current signature and vibration analysis}

There are numerous studied of WECS fault detection and diagnosis in the literature that could be classified as model-based approaches and data driven approaches. Vibration signal based CM have been commercially utilized in most of the WECS available, however the Accuracy and effectiveness of this method are affected by the sensor location and easily contaminated by environmental noise. Current based monitoring technique with vibration analysis reduces the requirement of Sensors and can be an effective approach for CM of WT. the parameters used in CSA are rotor current and stator current. The Figure 1 shows the schematic of the prognosis CM system that consist of several functional modules including signal conditioning, fault feature extraction, fault diagnosis and prognosis, RUL prediction, alarm management and equipment management. 


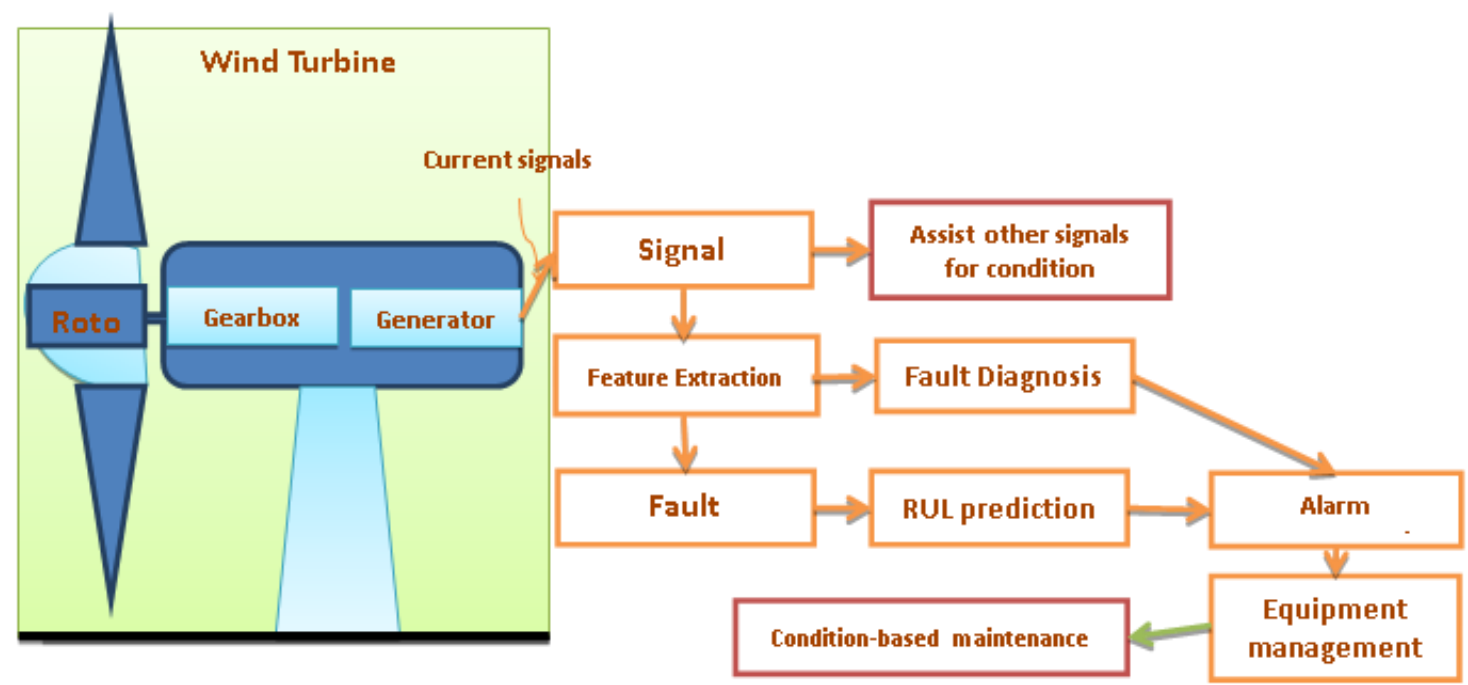

Figure 1. Schematic of a current based prognostic CM for WT

\subsection{Current signature analysis}

Fault in WT drive train component induces vibrations of the shaft at certain frequencies called fault characteristic frequencies in vibration and are proportional to the rotating frequencies of the shaft. This is a result of mechanical couplings between generator and failed drive train component(s), electromagnetic coupling between generator rotor and stator, this modulates the frequency and amplitude of generator stator/rotor current signals. A generator current signal i(t) can be expressed as follows:

$$
i(t)=\sum_{k} I_{k}(t) \sin \left\{2 \pi f_{k}(t) t+\varphi_{k}(t) \pm \sum_{j=1}^{M} I_{k, j}(t) \sin \left[\left[2 \pi f_{F, j}(t) t+\varphi_{k, j}(t)\right]\right\}\right.
$$

where $k$ is harmonic number, $I_{k}(t), f_{k}(t)$, and $\varphi_{k}(t)$ represent amplitude, frequency, and initial phase of the $k t h$ harmonic component, respectively; $I_{k, j}(t), f_{F, j}(t)$ and $\varphi_{k, j}(t)$ are the amplitude, frequency and initial phase of the $j t h$ fault characteristic frequency in vibration that modulates the frequency of the current signal, respectively. All the above values are time varying in nature.

Due to affect of amplitude modulation, the current signal can be expressed as follows:

$$
I_{k}(t)=I_{w, k}(t) \sum_{j=1}^{M} I_{k, j}(t) \sin \left[\int 2 \pi f_{F, j}(t) d t\right]
$$

Due to frequency modulation, each fault characteristic frequency in vibration $f_{F, j}(t)$ in (1) become an infinite number of sidebands around the harmonic frequency $f_{k}(t)$ in the current signal. It can be written as follows:

$$
i(t)=\sum_{k} I_{k}(t) \sin \left\{2 \pi\left[f_{k} \pm \sum_{j=1}^{M} \sum_{m} m f_{F, j}(t)\right] t+\varphi_{k}(t)\right\}
$$

Here $m$ is an integer indicating that the sidebands occur at multiples of faulty characteristic frequency in vibration $f_{F, j}(t)$ away from the harmonic frequency, $f_{k}(t)$. Above equations shows that the amplitude and frequency of the current signal both shows the information related to faults. Hence depending upon the location and amplitude of the fault characteristics frequency components extracted from the current signal, fault diagnosis can be performed. In practice only $k=1$ is considered for prognosis purpose. The current signal can also be assisted with vibration signals for CM of WT drive train under non-stationary operating conditions [9]. A most frequently used signal processing scheme is the FFT analysis of the current signal which gives the information of the fault characteristic frequencies. Variation of certain harmonic components in the frequency spectrum of the signal can be related to a specific fault and can be used as the fault signature for fault diagnosis of WT. Classical FFT is not capable of acquiring the information stored in a non-stationary signal of a WT. In such scenario the spectra of wavelet coefficient are analyzed in a specific frequency range that contains the features closely related to certain fault. Handling the computations with the FFT and power spectrum, it will be easy to understand the influence of windows on the spectrum. Several FFT-based functions that are extremely useful for network analysis can be done as shown in Figure 2. 


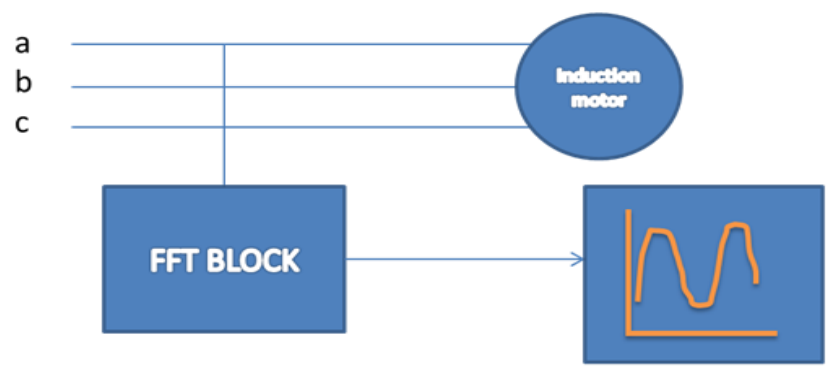

Spectrum analyser

Figure 2. FFT block to acquire frequency spectrum

\subsection{Vibrational analysis}

As said earlier vibration signal based CM is one of the matured CM schemes implied dominantly by commercially available WT. This technique has been standardized by ISO10816. This standard gives the guidelines for the measurements and classification of mechanical vibrations of reciprocating compressor system. Vibration sensors types majorly includes accelerometers, velocity sensors, and displacement sensors, with accelerometer having the widest working frequency range from 1 to $30 \mathrm{kHz}$, whereas the velocity sensors has working range from 10 to $1 \mathrm{kHz}$. Displacement sensors have a working frequency range from 1 to $100 \mathrm{~Hz}$. Due to large frequency range offered by accelerometer sensors, they are most widely used in CM of WT components. Vibration signals amplitude can be used to indicate the severity of the fault. The vibration sensors should be mounted on the surface or embedded in the body of the wind turbine. The vibration signals analysis for fault diagnosis can be done in three domains namely, time-frequency domain, time domain, and frequency domain. The vibration analysis tends to have low NSR and hence this can be improved by Resonance demodulation technology, Cepstrum analysis, and time domain average method. Accelerometer is the most widely used vibration sensor because of the wide frequency range they offer. They are manufactured to monitor gearbox, tower sway, and seismic motion in WT.

\section{PROPOSED CONDITION MONITORING SYSTEM}

\subsection{CSA aided vibration analysis $\mathrm{CM}$}

Till now we have just concentrated on a single WT but when we talk about wind parks or wind farms there is a group of WT in the same location. These Wind park have high installed capacity associated to them. When we talk about such a large number of WT and their condition monitoring wireless sensor network comes into picture. For wind park multiple WT CM schemes need to work together to give general idea about the health indication. In this phase we have concentrated on a single WT which can be expanded to a wind park. Data acquisition is a very crucial part of CM of WECS. For CSA data is acquired in the form of current signals from the generator in WT. This is done with the help of current transformer or other data acquisition techniques. For vibration analysis a range of vibration sensors are available dedicated for WT There is other vibration sensor available commercially for their application at different locations. Data pre-processing refers to the transfer of raw data to organised format to transform it in a useful format. This step includes data cleaning, data arrangement, data transformation, and data reduction.

The first step is data cleaning, as the data available may have many missing part or irrelevant information. This step involves handling of such noisy and missing data. We can handle the missing value in two ways, one is by ignoring the tuples, the other by filling the missing values. To handle the noisy data due to false data collection we can use three ways namely, binning method, regression or clustering. The second step is data transformation which is taken in order to transform data in appropriate form suitable for mining process. This can be done by normalization, attribute selection, discretization or concept hierarchy generation. The final step is Data reduction that is used to handle huge amount of data. This aims to increase the storage efficiency and reduce data storage and analysis cost and time. This can be achieved by data cube aggregation, attribute subset selection, numerosity reduction or dimensionality reduction. Feature selection is a very crucial step in machine learning algorithm, the features used for the training the data has a huge influence on the performance of your model. In this step either manually or by some algorithm selection of the most useful feature is done that contributes most to the prediction required for the system. This has a great effect on the accuracy and reliability of your prediction system 


\subsection{Simulation model development}

The parameter used for the generator model is given in Table 1 and the parameters of the gear box model is given in Table 2. A detailed model of the gearbox components is needed to acquire the dynamical behavior and to predict the loading conditions of the system as shown in Figure 3. To understand the development and exploitation of complex systems, approaches, tools that can provide simplified representations of reality is required. These simplified visions are called models which are based on graphical languages as shown in Figure 4. The wind turbine power curve is a graph that indicates how large the output will be for the turbine at different wind speeds is shown in Figure 5.

Table 1. Generator model parameters

\begin{tabular}{cc}
\hline Parameters & Values \\
\hline Nominal power & $1.65 \mathrm{MVA}$ \\
Voltage & $600 \mathrm{~V}$ \\
Frequency & $50 \mathrm{~Hz}$ \\
Stator resistance & $0.004843 \mathrm{Ohm}$ \\
Stator inductance & $0.01248 \mathrm{H}$ \\
Rotor resistance & $0.004377 \mathrm{ohm}$ \\
Rotor inductance & $0.1791 \mathrm{H}$ \\
No of poles & 6 \\
\hline
\end{tabular}

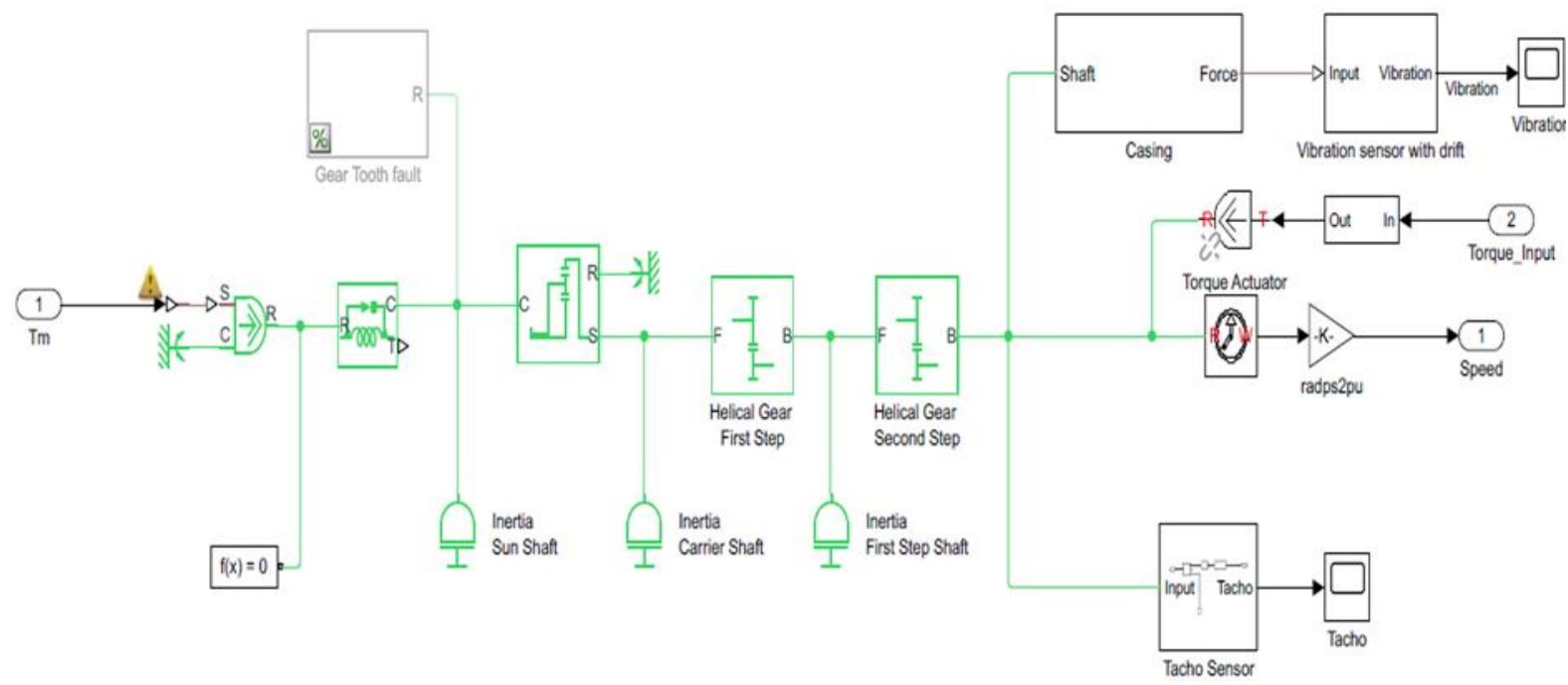

Figure 3. Gearbox model

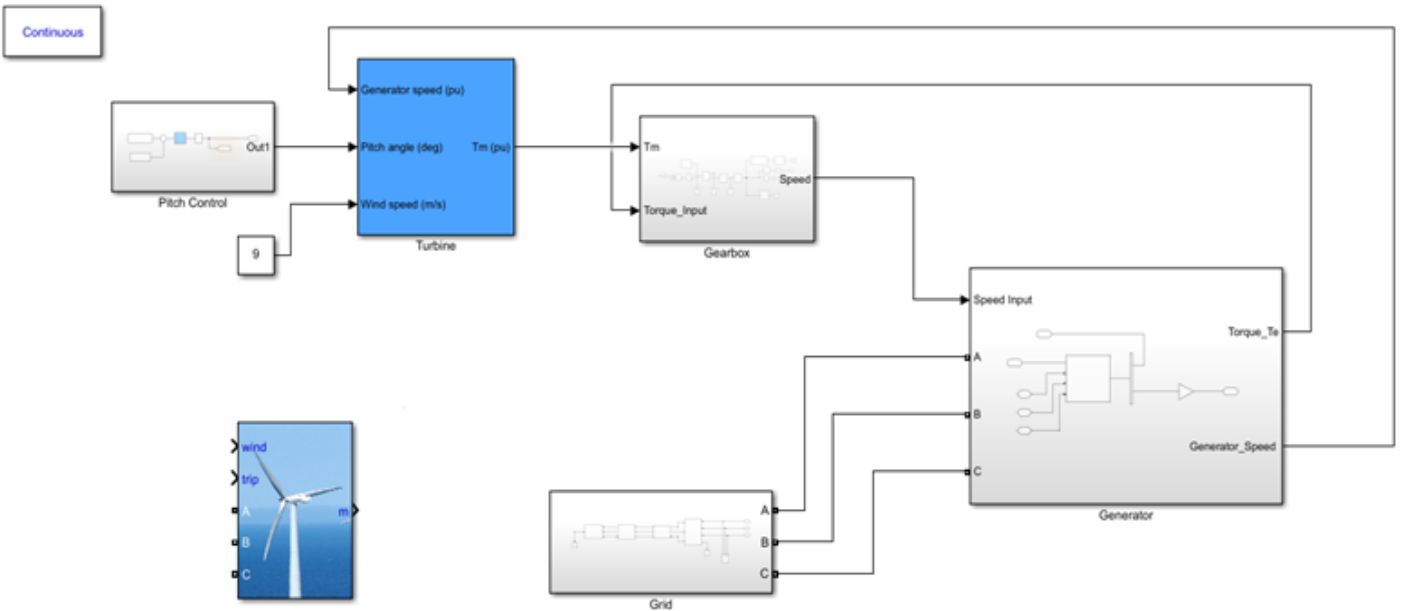

Figure 4. Block diagram for WT model
Table 2. Gearbox model parameters

\begin{tabular}{cc}
\hline Parameters & Values \\
\hline Inertia carrier shaft & 10 \\
Inertia sun shaft & 10 \\
Inertia first step shaft & 10 \\
Gear ratio first step & 4 \\
Gear ration Planetary & 5 \\
\hline
\end{tabular}




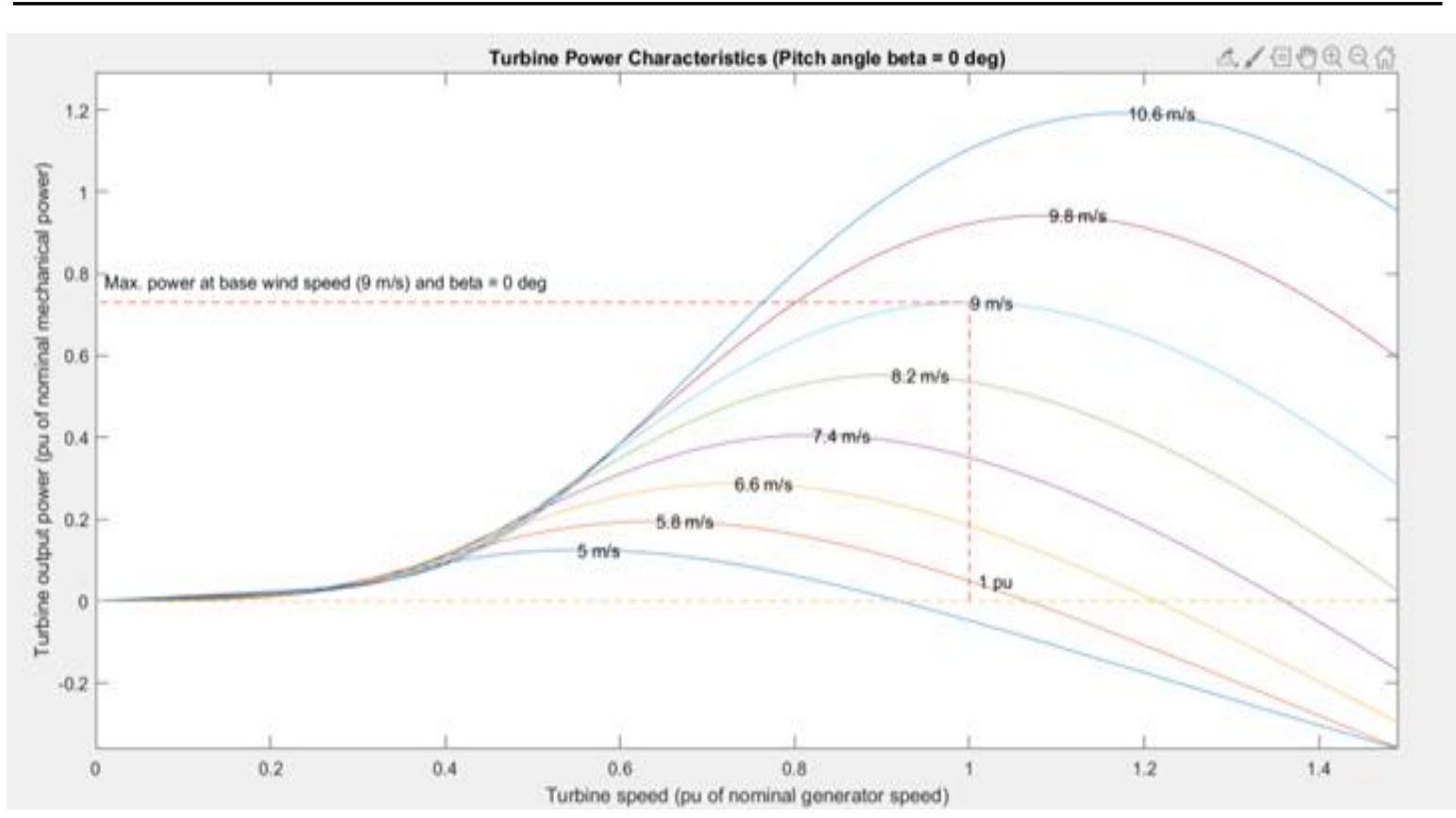

Figure 5. WT power characteristics

\subsubsection{Case study and discussions}

The dataset is collected from a $2 \mathrm{MW}$ wind turbine high-speed shaft driven by a 20 -tooth pinion gear. Vibration signal of 6 seconds was acquired each day for 50 consecutive days during the period an inner race fault developed and caused the failure of the bearing. In Table 3, 9 data from the existing data set is shown for the reference.

Table 3. Data collected from tachometer and data collected from vibration sensor

\begin{tabular}{cccc}
\hline No. & Technical Data & No. & Vibration Data \\
\hline 1 & 0.007383 & 1 & 0.178114 \\
2 & 0.007383 & 2 & 1.728471 \\
3 & 0.007383 & 3 & 1.039679 \\
4 & 0.007383 & 4 & -0.54949 \\
5 & 0.007383 & 5 & -0.87209 \\
6 & 0.007383 & 6 & -1.804 \\
7 & 0.007383 & 7 & -1.54984 \\
8 & 0.007383 & 8 & 0.255948 \\
9 & 0.007383 & 9 & 1.296981 \\
\hline
\end{tabular}

Extract the date information as independent variables so that we can make the data useful, and divide the data as per date and time. Now for Data Exploration and plotting, First visualize the vibration signals in the time domain. In this dataset, there are 50 vibration signals of 6 seconds measured in 50 consecutive days, which gives the following plot as shown in Figure 6 with the 50 vibration signals. Vibration signal in time domain shows increasing trend of signal impulsiveness. Spectral Kurtosis is considered as a powerful indicator in frequency domain for WT prognosis; hence information about it can help with the CM. The window size is taken as 128 . Color bar is used to indicate fault severity the range is from 0 to 1 . The Figure 7 shows that the spectral kurtosis value around $10 \mathrm{kHz}$ gradually increased as the machine condition degrades. 


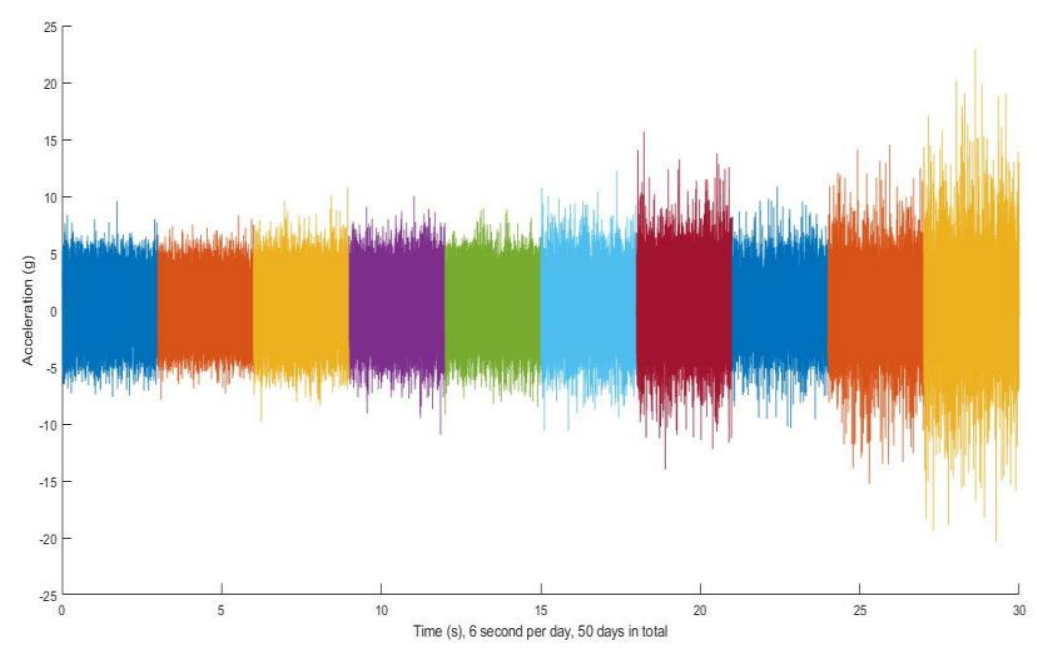

Figure 6. Vibration signals in the time domain

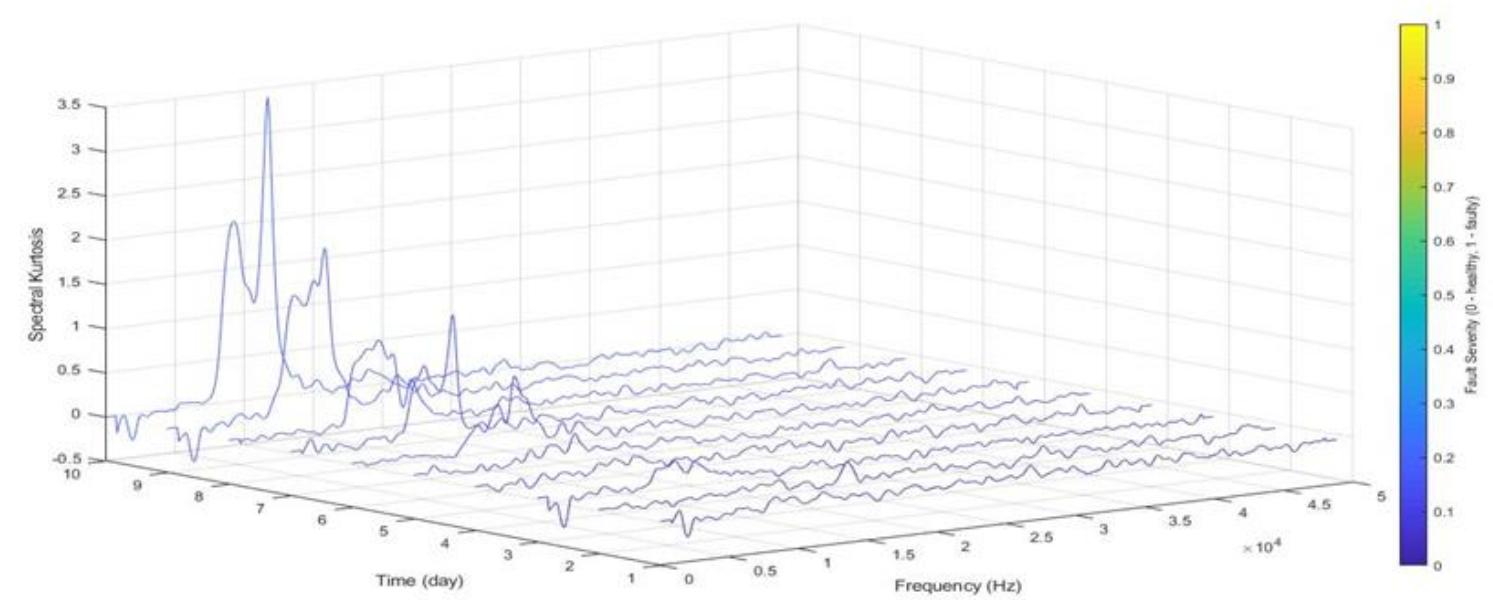

Figure 7. Spectral kurtosis

\subsubsection{Feature extraction}

Based on the above section analysis, statistical features from the time domain signal and spectral kurtosis can be derived that can be used as our fault feature for training purpose. The mathematical features include mean, peak to peak, crest factor, impulse factor, margin factor, energy, skewness, RMS and similarly for spectral kurtosis. These features are extracted with the available MATLAB functions. A feature table with all the data organised is created so that all the feature with their associated values with their time and date is created. The Table 4 shows the feature table for 4 days and 5 features.

Table 4. Feature table for 4 days and 5

\begin{tabular}{|c|c|c|c|c|c|c|}
\hline Date & Mean & Std & Skewness & Kurtosis & Peak2Peak & RMS \\
\hline '11-Mar-2013 03:00:24' & 0.213902311 & 2.088998915 & 0.006579094 & 3.04052075 & 21.216959 & 2.099918039 \\
\hline '16-Mar-2013 06:56:43' & 0.232812554 & 1.975499553 & -0.006068651 & 3.006899646 & 17.33607101 & 1.989167375 \\
\hline '21-Mar-2013 00:33:14' & 0.188993978 & 2.185210314 & 0.000366998 & 3.141638287 & 24.8842411 & 2.193364206 \\
\hline '26-Mar-2013 01:41:50' & 0.257405783 & 2.22928624 & 0.004230466 & 3.097450444 & 23.71159935 & 2.244094007 \\
\hline
\end{tabular}


When the smoothing parameters are smaller, the result will have less smoothed feature and Hence, the choice of the parameters need to align with required applications as shown in Figure 8. The indicated severity of the fault is measured and normalized into 0 to 1 scale. Then the spectral kurtosis value is fixed around $10 \mathrm{kHz}$ gradually increases in Figure 9. The peak2peak values are indicated before and after the smoothening in represented in Figure 10.
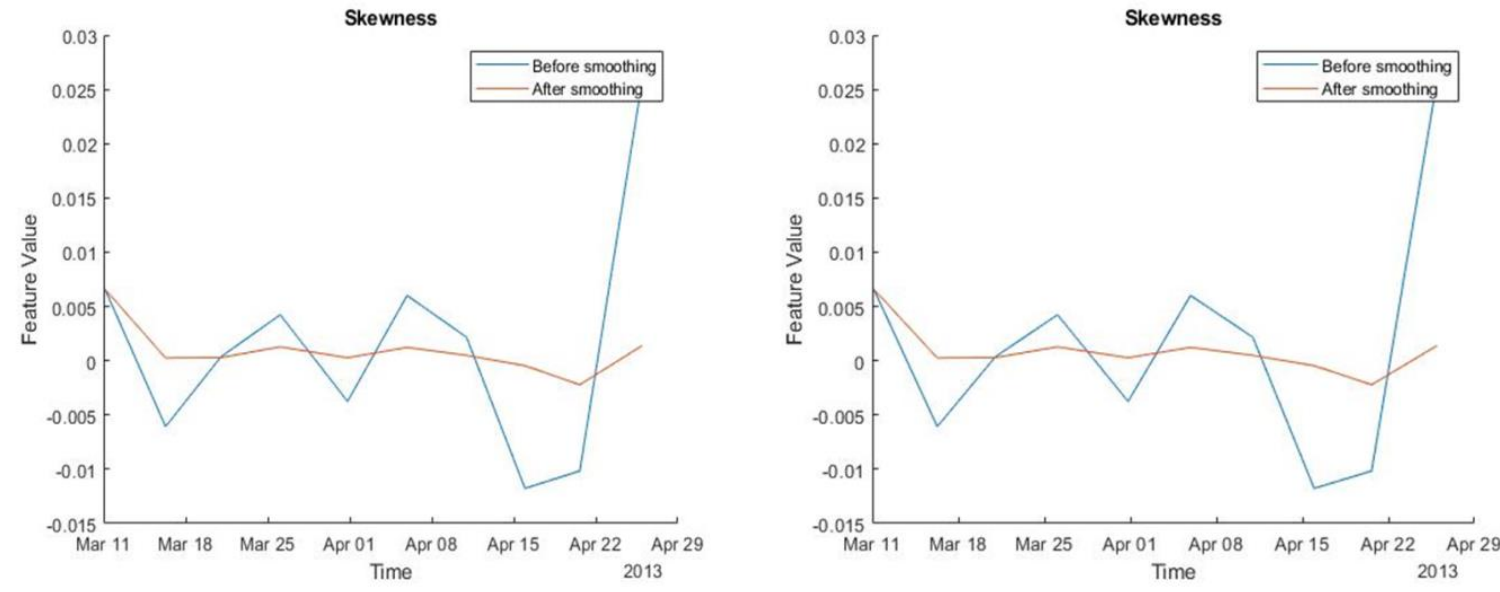

Figure 8. Standard feature smoothing

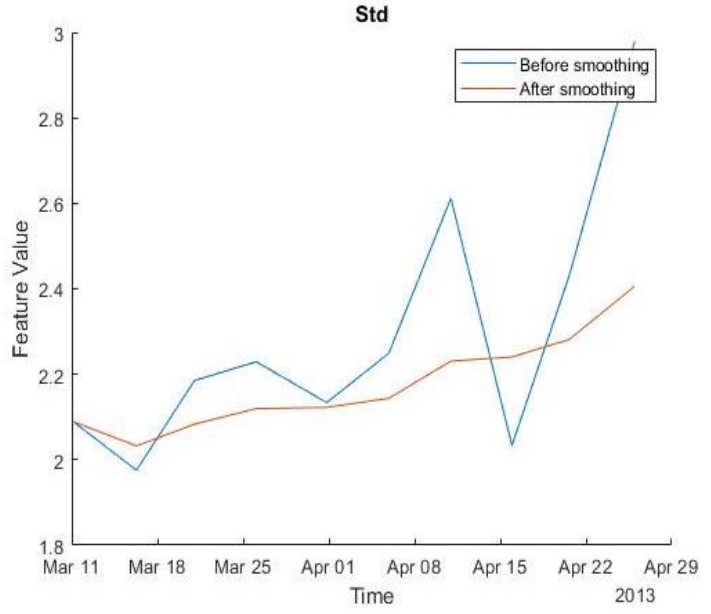

Figure 9. Kurtosis feature smoothing

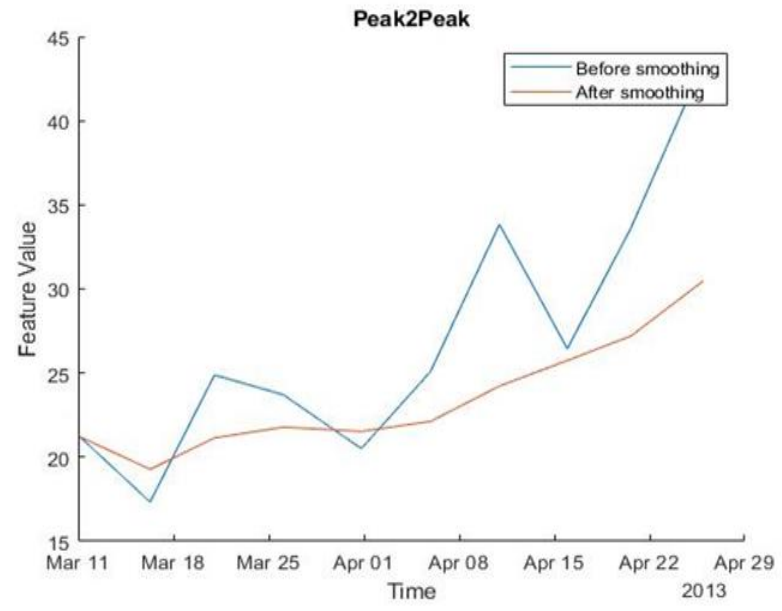

Figure 10. Peak2peak feature smoothing

\subsubsection{Fault prognosis}

Feature ranking is done using monotonicity, to select the best feature for training the data.

Monotocity $\left(x_{i}\right)=\frac{1}{m} \sum_{j=1}^{m} \frac{\left.\mid \text { number of positive diff }\left(x_{i}^{j}\right)-\text { number of negative diff } x_{i}^{j}\right) \mid}{n-1}$

Here, $n$ is number of measurement points, in this case $\mathrm{n}$ is $50, m$ is the number of machine monitored, in this case its $1 . x_{i}^{j}$ is the $i$ th feature measured on $j$ th machine. Kurtosis of the signal appears to be the top feature based on monotonicity. Figure 11 indicates that the first principal component is increasing as the machine approaches failure. Hence it is a promising health indicator. The health indicator is shown in Figure 12. 


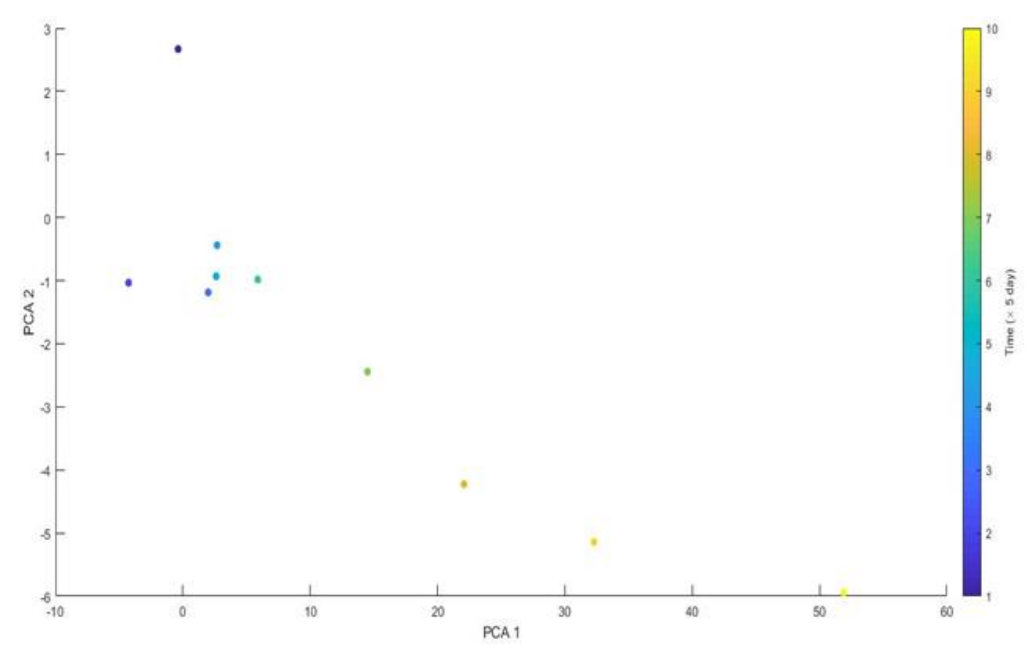

Figure 11. Plot for principal component

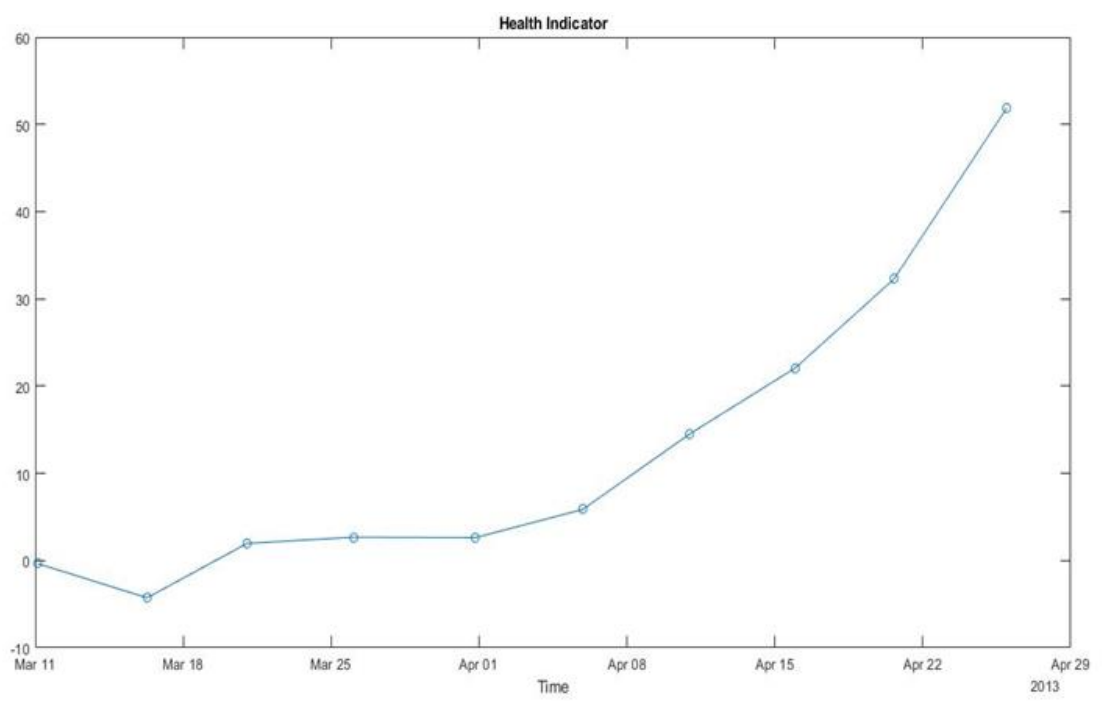

Figure 12. Plot for health indicator

\section{CONCLUSION}

From the work we can say that the vibration analysis alone is not enough for the affective CM of wind turbine and hence needs and aiding scheme. Internal factors of a WECS play a major role in designing the CM system for a WT. CM scheme is going to be different for every component and subsystem. The location of the sensor and its condition also plays a important role in the CM of WECS. If the complexity of the model is increased a better diagnostics approach can be developed which can be implemented in the real world with ease. The diagnostics approach should be applied to large scale and small scale WT. The work is done for a single WT which should be extended to a number of WT to get a reliable scheme for the CM of a wind park. More case studies should be done to get a reliable scheme for CM that is effective for onshore and offshore application of Wind Park.

\section{REFERENCES}

[1] X. Liu, Z. Gao and M. Z. Q. Chen, "Takagi-Sugeno Fuzzy Model Based Fault Estimation and Signal Compensation With Application to Wind Turbines," in IEEE Transactions on Industrial Electronics, vol. 64, no. 7, pp. 5678-5689, 2017. doi: 10.1109/TIE.2017.2677327

[2] L. Zhang and Z. Lang, "Wavelet Energy Transmissibility Function and Its Application to Wind Turbine Bearing Condition Monitoring," in IEEE Transactions on Sustainable Energy, vol. 9, no. 4, pp. 1833-1843, 2018. doi: 10.1109/TSTE.2018.2816738. 
[3] S. Djurovic, S. Williamson and A. Renfrew, "Dynamic model for doubly-fed induction generators with unbalanced excitation, both with and without winding faults," in IET Electric Power Applications, vol. 3, no. 3, pp. 171-177, 2009. doi: 10.1049/iet-epa.2008.0054.

[4] R. K. Ibrahim, S. J. Watson, S. Djurović and C. J. Crabtree, "An Effective Approach for Rotor Electrical Asymmetry Detection in Wind Turbine DFIGs," in IEEE Transactions on Industrial Electronics, vol. 65, no. 11, pp. 8872-8881, 2018. doi: 10.1109/TIE.2018.2811373.

[5] F. Cheng, L. Qu and W. Qiao, "Fault Prognosis and Remaining Useful Life Prediction of Wind Turbine Gearboxes Using Current Signal Analysis," in IEEE Transactions on Sustainable Energy, vol. 9, no. 1, pp. 157-167, 2018. doi: 10.1109/TSTE.2017.2719626.

[6] U. Vargas and A. Ramirez, "Extended Harmonic Domain Model of a Wind Turbine Generator for Harmonic Transient Analysis," in IEEE Transactions on Power Delivery, vol. 31, no. 3, pp. 1360-1368, 2016. doi: 10.1109/TPWRD.2015.2499701.

[7] W. Qiao and L. Qu, "Prognostic condition monitoring for wind turbine drivetrains via generator current analysis," in Chinese Journal of Electrical Engineering, vol. 4, no. 3, pp. 80-89, 2018. doi: 10.23919/CJEE.2018.8471293.

[8] S. George Fernandez, K. Vijayakumar, P. Suresh, et al., "Reduction of Transient Voltages in Switches using Embedded Machine Learning," International Journal of Power Electronics and Drive Systems, vol. 11, no. 1, pp. 235-241, 2020.

[9] D. Zhang, L. Qian, B. Mao, C. Huang, B. Huang and Y. Si, "A Data-Driven Design for Fault Detection of Wind Turbines Using Random Forests and XGboost," in IEEE Access, vol. 6, pp. 21020-21031, 2018. doi: 10.1109/ACCESS.2018.2818678.

[10] A. Sapena-Bano, J. Burriel-Valencia, M. Pineda-Sanchez, R. Puche-Panadero and M. Riera-Guasp, "The Harmonic Order Tracking Analysis Method for the Fault Diagnosis in Induction Motors Under Time-Varying Conditions," in IEEE Transactions on Energy Conversion, vol. 32, no. 1, pp. 244-256, 2017. doi: 10.1109/TEC.2016.2626008.

[11] G.F. Savari, V. Krishnasamy, et al., "Optimal Charging Scheduling of Electric Vehicles in Micro Grids Using Priority Algorithms and Particle Swarm Optimization,” Mobile Networks and Applications, vol. 24, pp. 1835-1847, 2019.

[12] F. Cheng, J. Wang, L. Qu and W. Qiao, "Rotor-Current-Based Fault Diagnosis for DFIG Wind Turbine Drivetrain Gearboxes Using Frequency Analysis and a Deep Classifier," in IEEE Transactions on Industry Applications, vol. 54, no. 2, pp. 1062-1071, 2018. doi: 10.1109/TIA.2017.2773426.

[13] W. Qiao and D. Lu, "A Survey on Wind Turbine Condition Monitoring and Fault Diagnosis —Part II: Signals and Signal Processing Methods," in IEEE Transactions on Industrial Electronics, vol. 62, no. 10, pp. 6546-6557, 2015. doi: 10.1109/TIE.2015.2422394

[14] J. Ribrant and L. M. Bertling, "Survey of Failures in Wind Power Systems With Focus on Swedish Wind Power Plants During 1997-2005," in IEEE Transactions on Energy Conversion, vol. 22, no. 1, pp. 167-173, 2007. doi: 10.1109/TEC.2006.889614

[15] W. Qiao and D. Lu, "A Survey on Wind Turbine Condition Monitoring and Fault Diagnosis -Part I: Components and Subsystems," in IEEE Transactions on Industrial Electronics, vol. 62, no. 10, pp. 6536-6545, 2015. doi: 10.1109/TIE.2015.2422112.

[16] G.F Savari, Gourab Saha "Optimal placement of Distributed Generation in a Distribution system using Hybrid Big Brunch \& Big Crunch Algorithm," International Journal of Control Theory and Applications, vol. 9, no. 16, 2016.

[17] Shulian Yang, Wenhai Li and Canlin Wang, "The intelligent fault diagnosis of wind turbine gearbox based on artificial neural network," 2008 International Conference on Condition Monitoring and Diagnosis, Beijing, 2008, pp. 1327-1330. doi: 10.1109/CMD.2008.4580221.

[18] G.F Savari,Kunal Malik, Satyajit Dora and K.Vijayakumar "A New Symmetric \& Asymmetric MultilevelInverter Topology with Reduced Maximum Blocking Voltage Switches," Journal of Advanced Research in Dynamical and Control Systems, vol. 7, no. 11, 2018.

[19] Z. Wang, H. Wang and W. Liu, "Fault Diagnosis of Wind Turbine Gearbox Based on Vibration Data," 2018 IEEE 15th International Conference on e-Business Engineering (ICEBE), Xi'an, pp. 234-238, 2018. doi: 10.1109/ICEBE.2018.00045.

[20] Ajibola Akinrinde, Andrew Swanson, Remy Tiako, "Effect of Ferroresonance on Wind Turbine: Comparison of Atp/Emtp and Matlab/Simulink," Indonesian Journal of Electrical Engineering and Computer Science, vol. 14, no. 3, pp. 1581-1594, 2019.

[21] J. Zeng, D. Lu, Y. Zhao, Z. Zhang, W. Qiao and X. Gong, "Wind turbine fault detection and isolation using support vector machine and a residual-based method," 2013 American Control Conference, Washington, DC, pp. 36613666, 2013. doi: 10.1109/ACC.2013.6580398.

[22] Tasnim, S., Rahman, A., Oo, A.M.T. et al., "Autoencoder for wind power prediction," Renewables Wind, Water, and Solar," vol. 4, no. 1, pp. 1-11, 2017. https://doi.org/10.1186/s40807-017-0044.

[23] G. Jiang, P. Xie, H. He and J. Yan, "Wind Turbine Fault Detection Using a Denoising Autoencoder With Temporal Information," in IEEE/ASME Transactions on Mechatronics, vol. 23, no. 1, pp. 89-100, 2018, doi: 10.1109/TMECH.2017.2759301.

[24] P. F. Odgaard and K. E. Johnson, "Wind turbine fault detection and fault tolerant control - An enhanced benchmark challenge," 2013 American Control Conference, Washington, DC, 2013, pp. 4447-4452.

[25] P.F. Odgaard, J. Stoustrup, and M. Kinnaert, "Fault tolerant control of wind turbines- a benchmark model," IFAC Proceedings Volumes, vol. 42, no. 8, pp. 155-160, 2009. 